\title{
A Method of Illumination Effect Transfer between Images Using Color Transfer and Gradient Fusion
}

\author{
Yi Zhang, Tianhao Zhao, Zhipeng Mo, Wenbo Li* \\ School of Computer Software, Tianjin University, Tianjin, P.R.C. \\ E-mail: yizhang@tju.edu.cn
}

\begin{abstract}
Illumination plays a crucial role to determine the quality of an image especially in photography. However, illumination alteration is quite difficult to achieve with existing image composition techniques. This paper proposes an unsupervised illumination-transfer approach for altering the illumination effects of an image by transferring illumination effects from another. Our approach consists of three phases. Phase-one layers the target image to three luminosity-variant layers by a series of pre-processing and alpha matting; meanwhile the source image is layered accordingly. Then the layers of the source image are recolored respectively by casting the colors from the corresponding layers of the target image. In phase-two, the recolored source image is edited to seamlessly transit at the boundaries between the layers using gradient fusion technique. Finally, phase-three recolors the fused source image again to produce a similar illuminating image with the target image. Our approach is tested on a number of different scenarios and the experimental results show that our method works well to transfer illumination effects between images.
\end{abstract}

\section{INTRODUCTION}

With the prevalence of digital cameras, the needs for image editing and enhancement arise. Digital image composition has recently received much attention for better visual effects in images. The goal of image composition is to combine objects or other image elements from various image sources to create a seamless and realistic image [1]. In traditional scenarios, photographers carefully conceive the color composition of their images to convey desired color themes and artists often use a restricted palette to create expressive work, and can also enhance color contrasts locally to give a better sense of solidity and depth [2-5]. Besides tone and color adjustments, illumination editing is another frequently used operation due to the crucial role of illumination effect in the determination of image quality.

Unfortunately, editing illumination effects of an image is a painful experience. Powerful commercial software, such as Photoshop, does not explicitly support the illumination effect editing. Such software typically adjusts the brightness of the image in a global manner, resulting in unsatisfying and unnatural visual effects. Thus, the users may have to search for a set of effective operations to edit the illumination effect through a large number of trials.

Currently, there are some important works about color alternation in the field of image editing, including color transfer $[6,7]$, color grading $[8,9]$ and tone as well as color theme enhancement $[10,11]$. Color transfer is a kind of process of color alteration, which transfers the target image's color statistics to the source image, making the color characteristics of the source image accord with those of the target one [12]. Applications of color transfer are very effective in changing the image mode and enhancing the quality of images. Nevertheless, the domains of color transfer or recoloring methods are limited because it might produce unnatural results when the illumination of source image and target image is quite different. To our best knowledge, there are few researches concerning illumination editing when dealing with image color transfer.

In this paper, we present an image-based approach to transfer illumination effects from the target image to the source image. Our idea is illustrated in Fig. 1. The approach consists of three phases. In phase-one, we segment the target image into several luminosity layers through a series of preprocessing and alpha matting to emphasize the luminosity changes of the image illumination. Accordingly, we layer the source image and transfer color characteristics of each layer from the target image to the corresponding layer of the source image. In general, the direct layer-based color transfer causes excessive color-inconsistence along the boundaries of layers. To resolve the problem of color discontinuity, phase-two edits the color of source image by dividing the source image to equal-size patches and performing gradient fusion [13] in patches. After that, the color-inconsistence artifact is reduced while the luminosity contrasts between layers are preserved. Finally, phase-three recolors the luminosity template derived from the previous phase by transferring the global color characteristics of the target image to it. Note that our approach adopts recoloring method [8,9] twice, one is in phase-one within the segmented layer, the other one is in phase-three for the whole image. With our approach, users can automatically re-illuminate their photographs according to example images with similar scene, reducing the amount of hand-made artifacts.

The rest of the paper is organized as follows: A review of related studies is briefly described in Section II. In Section III, we describe the framework and technical details of our proposed approach. Evaluations of the proposed approach are summarized in Section IV. Finally, we conclude the paper in Section V.

\footnotetext{
* Corresponding author: wenboli@tju.edu.cn
} 

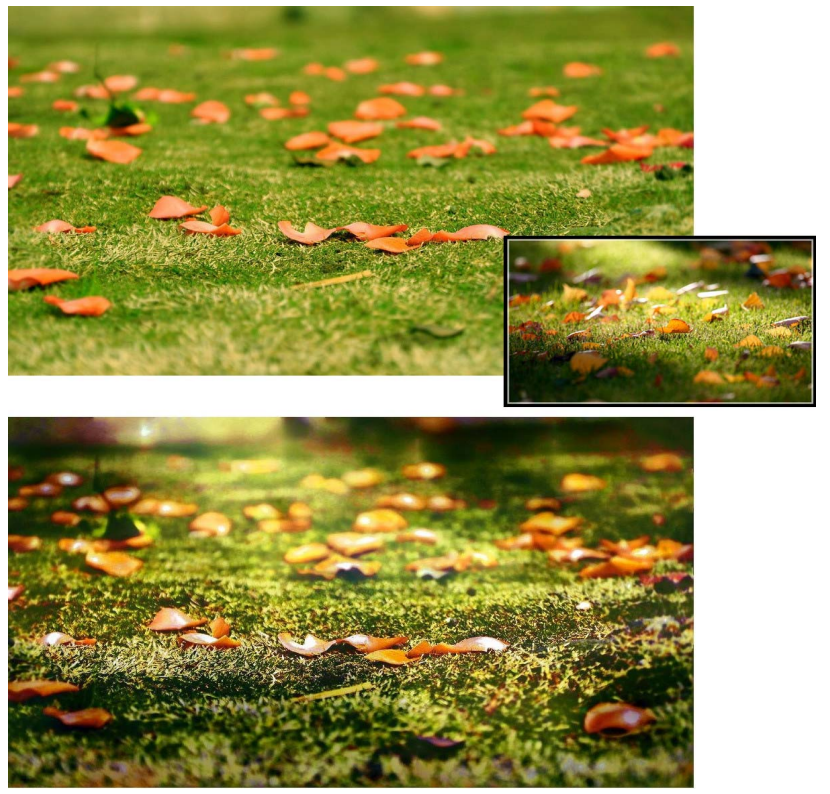

Fig. 1 Illumination transfer example. The source image on the top is reilluminated to the target image on the right-bottom, the result is showed on the bottom.

\section{RELATED WORK}

Digital image composition techniques are used to assemble existing image elements to create a seamless and convincing image. There have been many applications of image composition, such as image quality enhancement, image tone/theme enhancement, rapid prototyping design, etc. Among these applications, image quality enhancement and tone/theme enhancement are two most prevalent applications in our daily life. Color transfer techniques are typically used to realize the aforesaid enhancements.

\section{A. Color Transfer}

Color transfer algorithms aim to apply color characteristics including a color palette, tone or theme, from one image to another [5]. Reinhard et al. proposed the first popular color transfer method [6] which matches the mean and variance of the target image to the source image. The transfer of color statistics is performed separately on each channel in l $\alpha \beta$ color space [14]. Then their method has been extended to sequences in [15] and been further applied to video colorization in [16]. There are some researches considering possible dependence between channels. For instance, Ref. [17, 18] transfers the covariance information by aligning the principal component axes; Ref. [12] proposes an ellipsoid mapping scheme which extends Reinhard et al.'s work to correlated RGB color space. However, these color transfer techniques are limited to affine transformations of the color space. Thus some approaches to the non-linear mapping are proposed. A simple approach [19] is proposed to perform a histogram specification on the three color channels independently. Ref. [20] proposes a more complex mapping to deform tessellation meshes in the color space to fit to the $3 \mathrm{D}$ histogram of a uniform distribution.
Moreover, Pitié et al. [8, 9] proposed iterative color distribution transfer. This approach relies on applying a sequence of simple conversions with respect to randomly projected marginal color distributions instead of employing any explicit mapping function of the global color distribution. Specifically, the process of color transfer is to find a one-toone color mapping for each pixel in the original image. To realize this process, the author tried to find a mapping to transform the source color probability density function $(p d f) f$ into a new color $p d f$ which can match the $p d f g$ in the target image, that is:

$$
t(f)=g
$$

It treats the colors of an image as a distribution in a high dimensional space, and repeatedly projects this high dimensional distribution into a series of random 1D marginal distribution using the Radon Transform. The color distribution of the target image is converted to that of the source image by repeatedly mapping its 1D marginal distribution to those of the source image until convergence.

Apparently, most color transfer algorithms are concerned about the direct position-unrelated color transferring. Hence previous color transfer algorithms would be powerless when they try to meet the requirements depicted by Fig. 1. For this reason, we propose our approach. However, positioncorrelated methods would produce sharp color-inconsistence. To resolve this problem, we adopt gradient fusion technique.

\section{B. Gradient Fusion}

There have been many powerful approaches [21, 22] for seamless image composition. In this paper, we incorporate a simple gradient fusion method [13] proposed by Pérez et al. to our approach to modify the artifacts derived from the position-related color transfer method. The algorithm works by solving a set of Poisson equations that enforce the preservation of image gradient inside the inserted object and the pixel values along the boundaries. In this way, the colorinconsistence on the boundary is eliminated.

\section{PROPOSED APPROACH}

We describe our approach which incorporates the positioncorrelated color-transfer techniques and gradient fusion method to achieve image-based illumination composition in the following section.

\section{A. Overview of Our Approach}

The major difference between our work and other existing image composition methods is that we are concerned with position-related illumination transfer effect between images. Generally, the task of the proposed approach can be divided into three phases. As depicted in Fig. 2, each phase contains some critical components. For phase-one, once a target image and a source image are submitted to the system, the system first segments the illumination layers of the target image and thus generates a Matting Mask which restricts the process of layer-based color transfer later on. After transferring colors 
within each layer, the system outputs an illumination framework for the following phases. Next, phase-two divides the illumination framework into patches and enforces the preservation of the image gradients to eliminate the colorinconsistence caused by the layer-based color transfer. The luminosity template obtained in phase-two is then recolored globally in the phase-three and we obtain the image which has the similar illumination to the target image. The important notations for our approach have been presented in Table I.

\section{B. Phase One: Illumination Layer Model}

In our approach, we use $I_{o}$ and $I_{t}$ to denote the source image and the target image respectively. $I_{o}$ is the image to be reilluminated and $I_{t}$ is the image used as the example.

\section{Pre-processing}

The pro-processing of $I_{t}$ contains following three steps:

First, we divide $I_{t}$ into n square sections by $30 * 30$ pixels, denoted by $S_{1} \ldots S_{n}$. Assuming the pixel at position $(i, j)$ in $S_{m}$ $(1 \leq m \leq n)$ is defined as $P_{i, j}{ }^{m}$, where $1 \leq i \leq 30$ and $1 \leq j \leq 30$. Thus we have:

$$
\begin{aligned}
P_{\min }{ }^{m} & =\min \left(P_{1,1}{ }^{m} \ldots P_{1,30}{ }^{m} \ldots P_{30,1}{ }^{m} \ldots P_{30,30}{ }^{m}\right), \\
P_{\max }{ }^{m} & =\max \left(P_{1,1}{ }^{m} \ldots P_{1,30}{ }^{m} \ldots P_{30,1}{ }^{m} \ldots P_{30,30}{ }^{m}\right) .
\end{aligned}
$$

Then $I_{t}$ is converted to a gray-level image denoted by $I_{t \text {-gray }}$ and is normalized each pixel within the section of $I_{\text {t-gray }}$. The max-normalized $I_{t \text {-gray }}$ and min-normalized $I_{t \text {-gray }}$, denoted by $I_{t}^{\max }$ and $I_{t}^{\min }$ respectively, are constructed by replacing the values of pixels in each section with their maximum and minimum computed by Eq. (2). Furthermore, we extend $I_{t}^{\max }$ to the 3-channel image denoted by $I_{t 3}{ }^{\max }$ and $I_{t}^{\min }$ to the 3 channel image denoted by $I_{t 3}{ }^{\mathrm{min}}$.

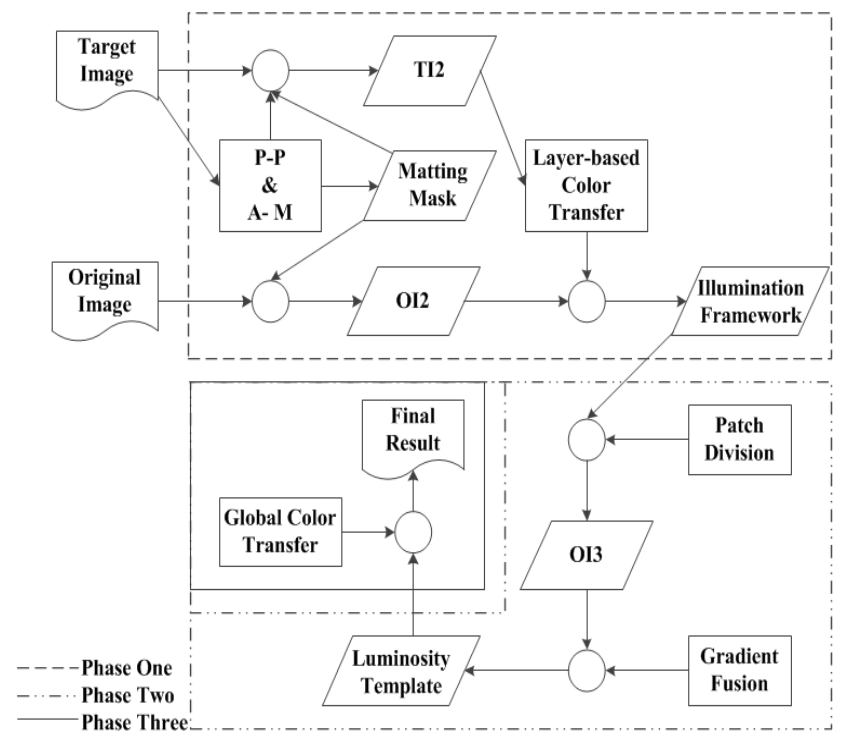

Fig. 2 Workflow of our approach. The proposed approach consists of three phases, and each phase contains some critical components. Note: The parallelograms (T12, OI2 and OI3) denote the intermediates and the circles denote the collaborations.
TABLE I

\begin{tabular}{|c|c|}
\hline Symbol & Meaning \\
\hline$I_{o}$ & Source image. \\
\hline$I_{t}$ & Target image. \\
\hline$I_{\text {t-gray }}$ & Gray level image of $I_{t}$ \\
\hline$S_{m}(m=1, \ldots, n)$ & $I_{t}$ is divided into $\mathrm{n}$ sections \\
\hline$P_{i, j}{ }^{m}$ & Pixel in $S_{m}(1 \leq i \leq 30,1 \leq j \leq 30)$ \\
\hline$I_{t}^{\max }$ & max-normalized $I_{t \text {-gray }}$ \\
\hline$I_{t}^{\min }$ & min-normalized $I_{\text {t-gray }}$ \\
\hline$I_{t 3}{ }^{\max }$ & A 3-channel image extended from $I_{t}^{\max }$ \\
\hline$I_{t 3}{ }^{\min }$ & A 3-channel image extended from $I_{t}^{\min }$ \\
\hline$R L$ & The layer with the highest illumination intensity in $I_{t}$ \\
\hline$Y L$ & The layer with less illumination intensity in $I_{t}$ \\
\hline$G L$ & The layer with the least illumination intensity in $I_{t}$ \\
\hline$I_{f}$ & Illumination framework \\
\hline$I_{g}$ & Global-recolored image of $I_{o}$ for $I_{t}$ \\
\hline$I_{l-t}$ & Luminosity template \\
\hline
\end{tabular}

NOTATIONS FOR OUR APPROACH

\section{Alpha Matting}

We employ alpha matting to segment the image. In our approach, the inputs to alpha matting are the image pair of $I_{t}$ and $I_{t 3}{ }^{\text {max }}$ and the image pair of $I_{t}$ and $I_{t 3}{ }^{\text {min }}$. Then it outputs Matting Mask as illustrated in Fig. 3: the red layer $(R L)$ represents the layer with the highest illumination intensity in $I_{t}$ while the yellow layer $(Y L)$ has less illumination intensity and the green layer $(G L)$ has the least illumination. Notably, $R L$ marks the matting result of the input pair of $I_{t}$ and $I_{t 3}{ }^{\text {min }}$ while $Y L$ marks the matting result of the input pair of $I_{t}$ and $I_{t 3}{ }^{\max }$. Furthermore, $I_{o}$ is arranged in layers according to Matting Mask.

\section{Layer-based Color Transfer}

The three layers in $I_{o}$ are recolored respectively using the colors of the corresponding layers in $I_{t}$. The recoloring result is an illumination framework $\left(I_{f}\right)$ which is presented as Fig. 5 (a). We summarize the algorithm for this step as follows:

\section{Algorithm 1 \\ Inputs: \\ - $I_{t}$ : Target image. \\ - $I_{o}$ : Source image.}

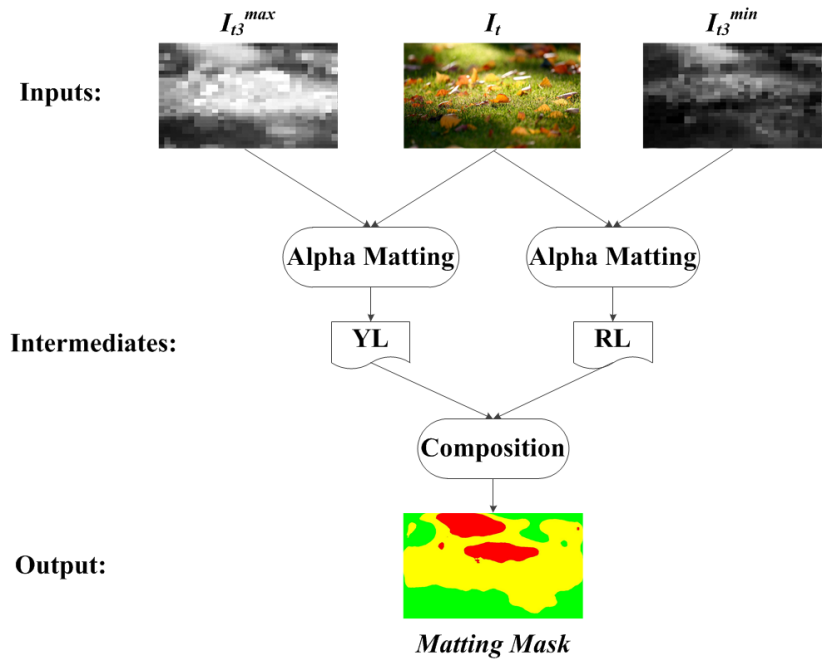

Fig. 3 Workflow of Matting Mask generation. The example image is the target image used in Fig. 1. 
- Matting Mask marked by $L_{i}(i=1: \mathrm{RL}, i=2$ : $\mathrm{YL}, i=3$ : GL).

\section{Output:}

- $I_{f}$. Luminosity framework.

\section{Processing:}

1) Initialize: $k \leftarrow 1$.

2) Iterate until $\mathrm{k}$ equals to 3 .

2.1)Map the 1D marginal distributions of $L_{i}$ in $I_{t}$ to that in $I_{o}$ until convergence.

2.2)Increase $k$ by 1 .

\section{Phase Two: Gradient Fusion}

After the layer-based color transfer, the illumination framework is color-inconsistent along the layers' boundaries. Inspired by [13], we use their approach to adjust the image color and Fig. 5 (b) shows one example result. First we divide the illumination framework $I_{f}$ and the global-recolored image $I_{g}$ into the same equal-size patches. In order to reserve the information of source image as much as possible, we divide $I_{g}$ into $5 * 5$ patches. Then the global-recolored patches are added to corresponding regions in illumination framework as inserted objects. By solving a set of Poisson equations in patches, the fusion result enforces the preservation of image gradient inside the inserted patches and the pixel values along the boundaries.

Gradient fusion is a guided interpolation method which can be used to eliminate the color-inconsistence between the neighboring regions. As illustrated in Fig. 4, we define the following notation:

- $S$ is the image definition domain (parts of $I_{f}$ in our case).

- $\Omega$ is a closed subset of $\mathrm{S}$ (a patch of $I_{f}$ in our case) with boundary $\partial \Omega$.

- $f^{*}$ is a known scalar function defined over $S$ minus the interior of $\Omega$.

- $f$ is an unknown scalar function defined over the interior of $\Omega$.

- $\quad \mathbf{v}$ is a vector field defined over $\Omega$.

- For each pixel $p$ in $S, N_{p}$ is the set of its 4-connected neighbors which are in $S . f_{p}$ is the value of $f$ at $p .<p$, $q>$ is the pixel pair with $q \in N_{p}$.

- The boundary of $\Omega$ is defined as $\partial \Omega=\left\{p \in S \backslash \Omega: N_{p}\right.$ $\cap \Omega \neq \varnothing\}$.

Our goal is to interpolate $I_{g}$ to $I_{f}$ by patches and numerically compute the set of intensities $\left.f\right|_{\Omega}=\left\{f_{p}, p \in \Omega\right\}$.

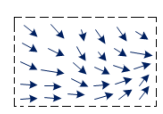

$\mathrm{V}$

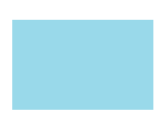

$g$

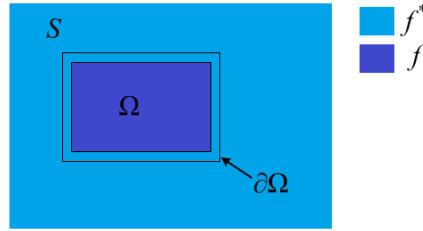

Fig. 4 Guided interpolation notations. Unknown function $f$ interpolates in domain $\Omega$ the destination function $f^{*}$, under guidance of vector field $\mathrm{v}$, which is the gradient field of a source function $g$ of a patch of $I_{g}$.
The interpolate $f$ of $f^{*}$ over $\Omega$ is defined as the solution of the following minimization problem:

$$
\min _{f \mid \Omega} \sum_{\langle p, q\rangle \Omega \neq \varnothing}\left(f_{p}-f_{q}-v_{p q}\right)^{2}, \text { with } f_{p}=f_{p}^{*} \text {, for all } p \in \partial \Omega,
$$

where $v_{p q}$ is the projection of $v\left(\frac{p+q}{2}\right)$ on the oriented edge $[p$, $q$ ], i.e., $v_{p q}=\mathrm{v}\left(\frac{p+q}{2}\right) \cdot \overrightarrow{p q}$. Its solution satisfies the following simultaneous linear equations:

for all $p \in \Omega,\left|N_{p}\right| f_{p}-\sum_{q \in N_{p} \Omega \Omega} f_{q}=\sum_{q \in N_{p} \cap \Omega} f_{q}^{*}+\sum_{q \in N_{p}} v_{p q}$.

Specifically, the basic choice for the guidance field $\mathrm{v}$ is a gradient field taken directly from a source image. Denoting by $g$ this source image, the interpolation is performed under the guidance of

$$
\mathrm{v}=\nabla g
$$

As for the numerical implementation, the continuous specification (5) translates into

$$
\text { for all }\left\langle p, q>, v_{p q}=g_{p}-g_{q}\right.
$$

which is to be plugged into (4).

The algorithm of this step is presented as follows:

\section{Algorithm 2 \\ Inputs:}

- $\quad P_{k}(k=1 \ldots 25)$ : patches of $I_{g}$.

- $I_{f}$

Output:

- $I_{l-t}$ : Luminosity template.

Processing:

1) Initialize: $k=1$;

2) Iterate until $k$ equals to 26 .

2.1)Iterate until pixels in $\Omega$ of $P_{k}$ are traversed.

2.1.1) Specify $N_{p}$ and compute $\sum_{q \in N_{p} \cap \partial \Omega} f_{q}^{*}$.

2.1.2) Construct the guidance field.

2.1.3) Travel to the adjacent pixel.

2.2)Construct the matrix by Eq. (4).

2.3)Solve the matrix.

2.4)Increase $k$ by 1 .

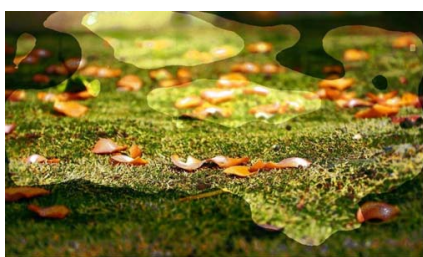

(a) Illumination Framework

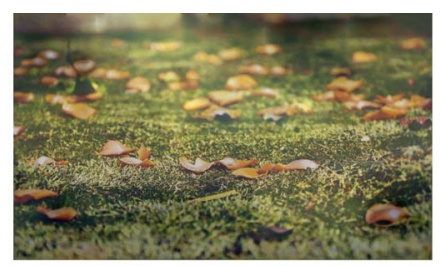

(b) Luminosity Template
Fig. 5 Illumination framework $\left(I_{f}\right)$ and luminosity template $\left(I_{l-t}\right)$ example. $I_{f}$ (a) and $I_{l-t}(\mathrm{~b})$ match the scenario illustrated in Fig. 1. 


\section{Phase Three: Luminosity Template Recoloring}

Empirically, $I_{l-t}$ preserves most luminosity information but loses color characteristics through gradient fusion. By applying recoloring to the image $I_{l-t}$, we transfer the color characteristics of $I_{t}$ to $I_{l-t}$, finally finish the task of modifying $I_{o}$ to be the image which has the similar illumination and color with the target image.

\section{EXPERIMENTAL RESULTS}

The proposed illumination transfer technique has been tested on a number of different scenarios. The results are presented in Fig. 6 and Fig. 7. In the experiments, we choose four groups of images. They are selected by two category partition criteria: natural illumination or artificial illumination, distant view or close view. The $1^{\text {st }}$ group is the still life under candle light; the $2^{\text {nd }}$ group is the skyline under sunlight; the $3^{\text {rd }}$ group is sea of clouds under sunlight and the $4^{\text {th }}$ group is the lawn under sunlight.

In HSV color space, channel V reflects the luminosity of an image. Therefore we use the L1 norm and standard deviation of each corresponding pixel pair in channel $\mathrm{V}$ between target image and re-illumination result image as evaluation measurements. Small L1 norm and standard deviation mean that the two images are more similar. We compare the results of our approach with the results of recoloring technique [8,9] in both visual effect and difference measurements. Fig. 6 shows the comparisons in visual effect and Table $\Pi$ shows the results comparison in the sum of L1 norm difference and the sum of standard deviation difference. We can tell from the Fig. 6 that the results of our method can reserve more illumination characteristic than recoloring method. The same conclusion can be drawn from Table $\Pi$. Compared with the pure recoloring result, our method is more close to the target image in pixel level.

\section{CONCLUSIONS}

This paper proposes a method for illumination transfer between images. The method is based on an illumination layer model which layers the illumination region in an image and transfers the color statistics to reconstruct the illumination effects. Then gradient fusion is employed to eliminate the color-inconsistence along the boundaries of the layers. The experimental results demonstrate that our method can achieve illumination transfer on the base of color transfer. However, our approach is position-related; hence it may fail when the source image and target image differ too much.

TABLE $\Pi$

DIFFERENCE EVALUATION MEASUREMENT AND COMPARISONS

\begin{tabular}{ccccc}
\hline Group & \multicolumn{2}{c}{ Sum of L1 Norm } & \multicolumn{2}{c}{ Sum of Standard Deviation } \\
& Recoloring & Our method & Recoloring & Our method \\
\hline $1^{\text {st }}$ & $1.0414 \mathrm{e}+04$ & $\mathbf{7 . 1 7 1 e + 0 3}$ & 75.8 & $\mathbf{4 9 . 8}$ \\
$2^{\text {nd }}$ & $1.0716 \mathrm{e}+04$ & $\mathbf{1 . 5 3 7 7 e + 0 4}$ & 75.8 & $\mathbf{5 3 . 4}$ \\
$3^{\text {rd }}$ & $1.7917 \mathrm{e}+04$ & $\mathbf{1 . 5 2 2 1 e}+\mathbf{0 4}$ & 74.4 & $\mathbf{6 4 . 1}$ \\
$4^{\text {th }}$ & $1.8291 \mathrm{e}+05$ & $\mathbf{1 . 2 5 2 3 e}+\mathbf{0 5}$ & 284.1 & $\mathbf{2 0 2 . 5}$ \\
\hline
\end{tabular}

\section{REFERENCES}

[1] Mortensen Eric N and Barrett, William A, "Intelligent scissors for image composition," in Proceedings of the 22nd annual conference on Computer graphics and interactive techniques, ed: ACM, 1995, pp. 191-198.

[2] Choi KD, Oil painting. Mijin Company, Seoul Korea, 1999.

[3] Luft Thomas and Colditz, Carsten and Deussen, Oliver, "Image enhancement by unsharp masking the depth buffer." vol. 25, ed: ACM, 2006.

[4] Quiller S, Color choices: making color sense out of color theory. Watson-Guptill, New York, 1989.

[5] Seo SangHyun and Park, YoungSub and Ostromoukhov, Victor, "Image recoloring using linear template mapping," in Multimedia Tools and Applications, ed: Springer, 2012, pp. 1-16.

[6] Reinhard Erik and Adhikhmin, Michael and Gooch, Bruce and Shirley, Peter, "Color transfer between images," in IEEE Computer Graphics and Applications. vol. 21, ed: IEEE, pp. 34-41.

[7] Chang Youngha and Saito, Suguru and Nakajima, Masayuki, "A framework for transfer colors based on the basic color categories," in Computer Graphics International, 2003. Proceedings, ed: IEEE, 2003, pp. 176-181.

[8] Pitie Francois and Kokaram, Anil C and Dahyot, Rozenn, "N-dimensional probability density function transfer and its application to color transfer," in Tenth IEEE International Conference on Computer Vision, 2005. ICCV 2005. vol. 2, ed: IEEE, 2005, pp. 1434-1439.

[9] Pitie Francois and Kokaram, Anil C and Dahyot, Rozenn, "Automated colour grading using colour distribution transfer," in Computer Vision and Image Understanding. vol. 107, ed: Elsevier, 2007, pp. 123--137.

[10] Wang Baoyuan and Yu, Yizhou and Xu, Ying-Qing, "Example-based image color and tone style enhancement," ACM Transactions on Graphics (TOG), vol. 30, p. 4, 2011.

[11] Wang Baoyuan and Yu, Yizhou and Wong, Tien-Tsin and Chen, Chun and $\mathrm{Xu}$, Ying-Qing, "Data-driven image color theme enhancement," in ACM Transactions on Graphics (TOG). vol. 29, ed: ACM, 2010, p. 146.

[12] Xiao Xuezhong and Ma, Lizhuang, "Color transfer in correlated color space," in Proceedings of the 2006 ACM international conference on Virtual reality continuum and its applications, ed: ACM, 2006, pp. 305-309.

[13] Perez Patric and Gangnet, Michel, "Poisson image editing," in ACM Transactions on Graphics (TOG). vol.22, ed: ACM, 2003, pp. 313-318.

[14] Ruderman Daniel L and Cronin, Thomas W and Chiao, Chuan-Chin, "Statistics of cone responses to natural images: Implications for visual coding," in JOSA A. vol. 15, ed: Optical Society of America, 1998, pp. 2036-2045.

[15] Wang Chung-Ming and Huang, Yao-Hsien, "A novel color transfer algorithm for image sequences," in Journal of Information Science and Engineering. vol. 20, ed: Citeseer, 2004, pp. 1039-1056. 
[16] Yatziv Liron and Sapiro, Guillermo, "Fast image and video colorization using chrominance blending," in IEEE Transactions on Image Processing. vol. 15, ed: IEEE, 2006, pp. 1120-1129.

[17] Abadpour Arash and Kasaei, Shohreh, "A fast and efficient fuzzy color transfer method," in Proceedings of the Fourth IEEE International Symposium on Signal Processing and Information Technology, 2004. , ed: IEEE, 2004, pp. 491-494.

[18] Kotera Hiroaki, "A scene-referred color transfer for pleasant imaging on display," in IEEE International Conference on Image Processing, 2005. ICIP 2005. vol. 2, ed: IEEE, 2005, pp. 5-8.

[19] Grundland Mark and Dodgson, Neil A, "Color histogram specification by histogram warping," in Electronic

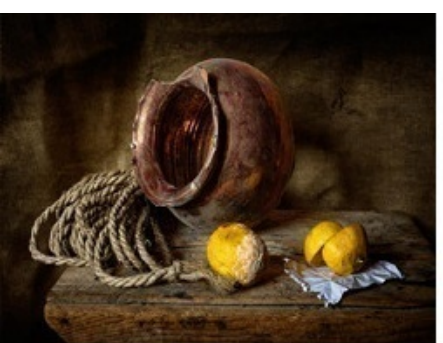

Group 1 : Source

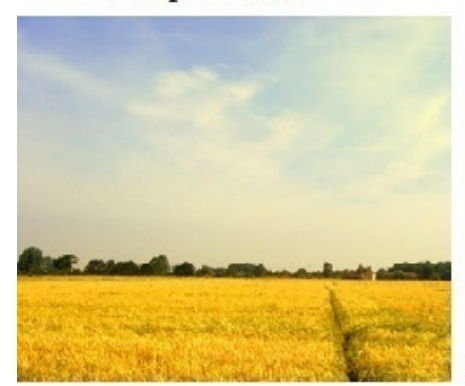

Group a : Source

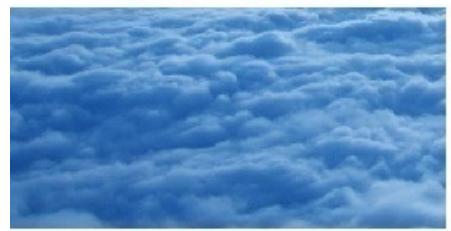

Group 3 : Source

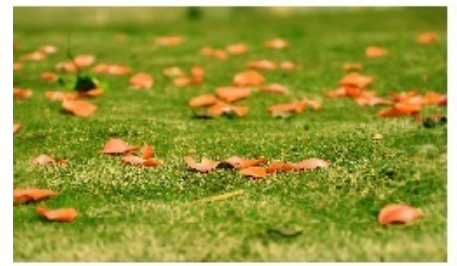

Group 4: Source

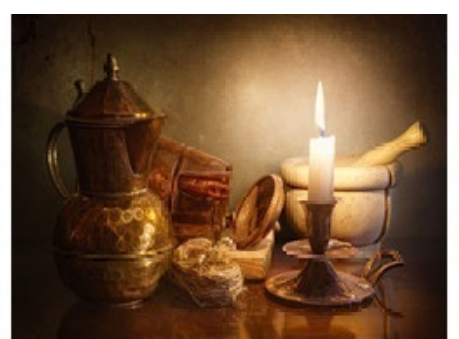

Target

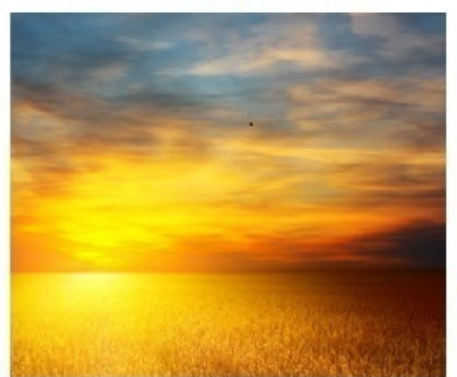

Target

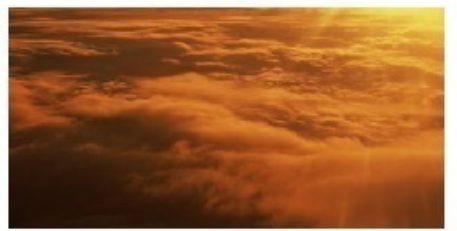

Target

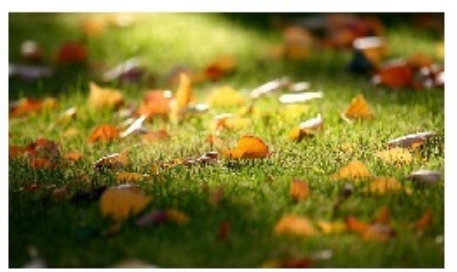

Target

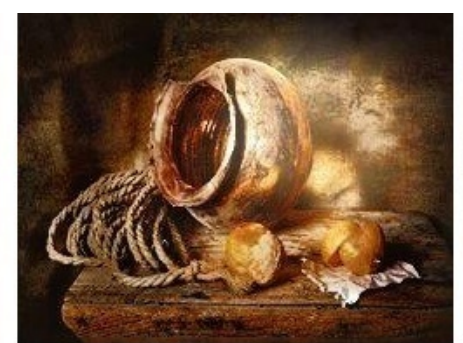

Our result

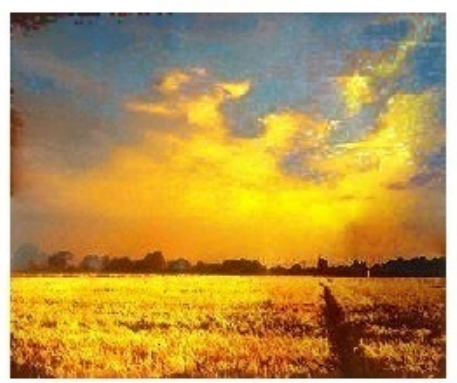

Our result

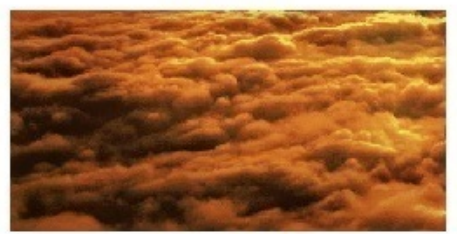

Our result

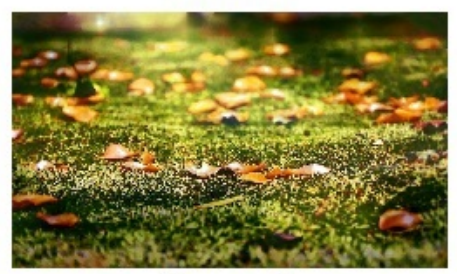

Our result

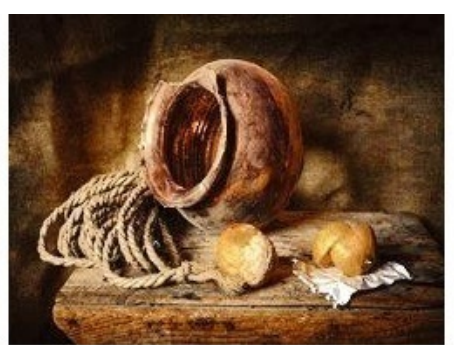

Result of color transfer

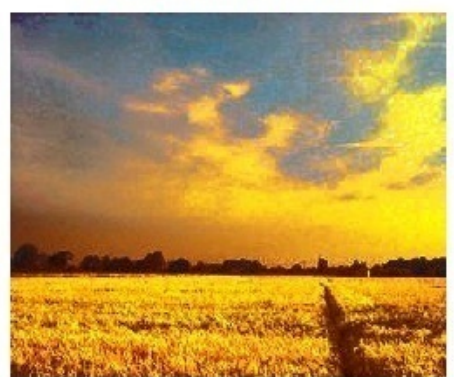

Result of color transfer

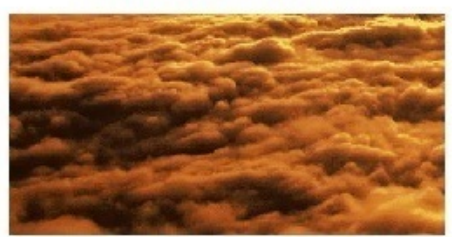

Result of color transfer

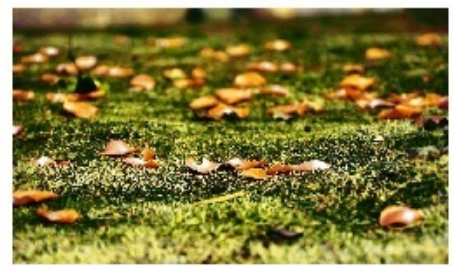

Result of color transfer

Fig. 6 Results of illumination transfer and comparisons with recoloring method [8,9] 